\title{
Characterization of spinal cord diffusion tensor imaging metrics in clinically asymptomatic pediatric subjects with incidental congenital lesions
}

\author{
Sona Saksena ${ }^{1} \cdot$ Mahdi Alizadeh $^{1,2} \cdot$ Devon M. Middleton $^{1} \cdot$ Chris J. Conklin $^{1} \cdot$ Laura Krisa $^{3} \cdot$ Adam Flanders $^{1} \cdot$ \\ MJ Mulcahey ${ }^{3} \cdot$ Feroze B. Mohamed ${ }^{1} \cdot$ Scott H. Faro ${ }^{4}$
}

Received: 3 December 2017 / Revised: 9 March 2018 / Accepted: 31 March 2018

(c) International Spinal Cord Society 2018

\begin{abstract}
Study design Retrospective study.

Objectives To perform quantitative DTI measurements of the entire cervical and thoracic spinal cord (SC) in typically developing (TD) pediatric subjects with incidental findings of syringomyelia or hydromyelia on conventional MRI and in a TD population without any abnormalities.

Setting USA.

Methods 26 TD recruited as part of large SC DTI study, four of these had incidental findings. Axial DTI images were acquired on 3T MR scanner to cover the cervical and thoracic SC. We performed group analysis of DTI values in the cord above and below the MR-defined lesion. For single-subject analysis, the cord above and below the lesion was compared to average values of TD population. A standard least squares regression model was used to compare DTI parameters fractional anisotropy (FA), mean diffusivity (MD), axial diffusivity (AD), and radial diffusivity (RD) between TD population and subjects with hydromyelia and syringomyelia. A $p$ value of 0.05 was used for statistical significance.

Results In group analysis, MD and AD were significantly different in cord above the lesion in subjects with hydromyelia and syringomyelia $(n=4)$ compared to TD population $(n=22)$. For single-subject analysis, DTI parameters were significantly different in cord above the syringomyelia and below the syringomyelia; $\mathrm{MD}, \mathrm{AD}$, and RD were significantly different. A subject with hydromyelia showed significant difference in FA below the lesion.

Conclusions This study demonstrates that DTI has the potential to be used as an imaging biomarker to evaluate SC above and below the congenital lesion in syringohydromyelia subjects.
\end{abstract}

\section{Introduction}

Syringomyelia and hydromyelia are abnormal fluid-filled cavitary benign lesions within the spinal cord (SC) that are

Sona Saksena

Sona.Saksena@jefferson.edu

1 Department of Radiology, Thomas Jefferson University, Philadelphia, PA, USA

2 Department of Neurosurgery, Thomas Jefferson University, Philadelphia, PA, USA

3 Department of Occupational Therapy, Thomas Jefferson University, Philadelphia, PA, USA

4 Department of Radiology, Johns Hopkins School of Medicine, Baltimore, MD, USA uncommon to rare [1,2]. Syringomyelia is characterized by a collection of cerebrospinal fluid in the intramedullary space within the SC [3, 4], while hydromyelia is described as a dilatation of the central canal and ependymal lined. In contradistinction to hydromyelia, syringomyelia is lined by glial cells [5]. Syringomyelia occurs in 8.4 per 100,000 individuals in the United States [6], however, the prevalence of syringomyelia in the general pediatric population is unknown [7]. In children, syringohydromyelia usually occurs in the setting of congenital anomalies, most commonly the Chiari-I malformation and tethered cord, but it can also develop years after meningitis and spinal trauma or with intramedullary and extramedullary tumors [8]. The estimated prevalence of syringomyelia associated with these conditions is between 1 in 1300 and 1 in 1900 respectively [9]. 
Diffusion tensor imaging (DTI) has been shown to assess the microstructural changes in patients with syringomyelia [10]. However, the prevalence of these abnormalities in the clinically normal pediatric population is uncommon to rare. Yan et al. described in a population of 23 patients, 8-25 years old, with syringomyelia, that fractional anisotropy (FA) values above and below the syringomyelia were not statistically different compared to normals [10]. In our study of 26 typically developing (TD) pediatric subjects scanned, $16 \%$ had unexpected incidental findings of syringomyelia or hydromyelia on conventional magnetic resonance imaging (MRI). These incidental findings were present in the thoracic SC and represented hydromyelia lesion in three subjects and syringomyelia lesion in one subject. These subjects with syringomyelia or hydromyelia had no known neurological deficits and were clinically normal. Syringomyelia and hydromyelia are fluid-filled cavitary benign lesions that do not cause disruption of the adjacent cord tissue. Therefore the cord above and below the congenital lesion, which appeared normal on MRI, would be expected to be normal on DTI maps. The purpose of this study was to perform quantitative DTI measurements along the length of the entire SC in these subjects and in a TD population. Both group analysis (combined hydromyelia and syringomyelia cases) and single-subject DTI analysis relative to the TD population average was performed.

\section{Methods}

\section{Subjects}

Out of 26 TD pediatric subjects recruited as part of large SC DTI study, four subjects (mean age, $11.51 \pm 3.62$ years) had incidental findings of hydromyelia and syringomyelia. The enrolled TD subjects were recruited in a consecutive manner. A few TD subjects were recruited as siblings of some of our spinal cord injury population and a few through our colleagues or family members. These four TD subjects had no known neurological deficits and no known etiology to explain hydromyelia and syringomyelia and therefore represented a congenital process. Subjects were included in the study if they successfully passed a brief neurological screening assessing motor and balance function. In addition, subjects were asked about their past medical history which included questions related to positive muscle, bone, or sensory findings. The subjects who were unable to tolerate MRI without sedation; had infantile, juvenile or adolescent idiopathic scoliosis or; any abnormality of the nervous and/or musculoskeletal system were excluded. Written informed assent (child) and consent (parent) were obtained under the protocol approved by Institutional review board.

\section{Data acquisition}

The scans were performed using a 3T Verio MR scanner (Siemens, Erlangen, Germany) with a 4-channel neck matrix and an 8-channel spine matrix coil. Conventional T1- and T2-weighted MRI scans were obtained for all the subjects. The T2-weighted image of the cervical and thoracic SC in the sagittal plane was initially obtained using a gradient echo (GRE) sequence and was used to facilitate slice prescription for the subsequent scans perpendicular to the cord. Next, an axial GRE T2-weighted, sagittal T2weighted 3D SPACE, sagittal turbo spin-echo (TSE) T1weighted, sagittal TSE T2-weighted, and axial DTI scans were obtained for the subjects.

DTI data collection was acquired using an inner field of view (iFOV) sequence. The iFOV sequence was based on a diffusion sensitized single-shot echo-planar imaging sequence using 2D radiofrequency excitations which allows for higher in-plane resolution with fewer geometric distortions and improves signal-to-noise ratio [11, 12]. Manual shim volume adjustments were also performed prior to data acquisition in order to restrict the adjustment volume to the anatomy of interest in an effort to further improve signal.

The iFOV sequence was optimized for both signal and scan duration to image the entire pediatric SC. High inplane resolution axial diffusion-weighted images were acquired in the same anatomical location prescribed for the T2-weighted GRE to cover the cervical (C1-upper thoracic region) and thoracic (upper thoracic-L1) SC using two overlapping slabs that ensured complete coverage [13]. The imaging parameters for each slab of DTI acquisition included: $\mathrm{FOV}=164 \mathrm{~mm}$, phase $\mathrm{FOV}=28.4 \%(47 \mathrm{~mm})$, three averages of 20 diffusion directions images were acquired in addition to $6 \mathrm{~b} 0$ images, $b$-value $=800 \mathrm{~s} / \mathrm{mm}^{2}$, voxel size $=0.8 \times 0.8 \times 6 \mathrm{~mm}^{3}$, axial slices $=40, \quad \mathrm{TR}=$ $7900 \mathrm{~ms}$, TE $=110 \mathrm{~ms}$, and acquisition time $=8: 49 \mathrm{~min}$. Anesthesia was not administered to the subjects in this study and cardiac/respiratory gating was not used to keep scan times as short as possible.

\section{Data processing}

A central mask was applied to the raw DTI images to eliminate the anatomy outside the SC. Motion correction was performed first on the six b0 images to create a mean b0 image, and the diffusion-weighted images were subsequently co-registered to the mean b0 image [14]. The diffusion data sets were corrected for motion-induced artifacts based on 3D registration technique using an in-house software developed in MATLAB (MathWorks, Natick, Massachusetts) [14]. The technique uses rigid body transformation with six degrees of freedom (three 
translational, three axis rotations) paired with normalized mutual information as cost function to align target images (20 diffusion directional images) with the reference image (b0). A robust, iterative diffusion tensor estimation scheme, RESTORE, was implemented to ensure removal of data outliers from the final tensor fit [15]. All the processing was performed using in-house software developed in MATLAB [13].

\section{Region of interest analysis}

After diffusion tensor estimation and generation of the diffusion maps, regions of interest (ROI) were drawn to extract information from the whole cord. Each ROI was manually drawn on grayscale FA maps at every axial slice based on the methodology from the previous paper along the cervical and thoracic SC [13]. For drawing the ROIs, slices corresponding to the anatomic regions with accurate vertebral levels were defined for the cervical and thoracic cord regions by a board-certified pediatric neuroradiologist. There was a consistent sparring of the outer margin of the cord of approximately one voxel width to minimize volume averaging with the cerebrospinal fluid. DTI parameters FA, mean diffusivity (MD), axial diffusivity ( $\mathrm{AD}$ ), and radial diffusivity (RD) were quantified at each intervertebral disk level and at the mid-vertebral body level of the cervical and thoracic SC (C1 to Mid L1) in all subjects.

In this study, the subjects with hydromyelia had lesion from T3-T4 to T5-T6 (subject 1), Mid T6 to T10-T11 (subject 2), and T7-T8 to Lower L1 (subject 3) while the subject with syringomyelia had their lesion from Mid T3 to T4-T5 (subject 4). In all the four subjects, the cord was normal appearing above the lesion identified on conventional MRI. This normal appearing cord above a lesion was defined by the level of the most superior MR-defined lesion, which was at Mid T3. Hence, for group analysis, mean values from $\mathrm{C} 1$ to $\mathrm{T} 2-\mathrm{T} 3$ levels of these four subjects were compared to the corresponding levels of the averaged TD population. A group analysis of the normal appearing cord below the lesion was also performed. Three of the four subjects (subjects 1, 2, and 4) had normal appearing cord below the lesion identified on MRI. The lowest lesion (subject 2) was at the T10-T11 level and therefore the cord below this lesion level (mid T11 to the conus) was used for the group analysis and was compared to the same levels of the mean TD population. Subject 3 had a lesion that extended to the conus at the L1 level and therefore had no cord inferior to the lesion. For singlesubject analysis, the cord above (subjects 1, 2, 3, 4) and below (subjects $1,2,4)$ the lesion was compared to the corresponding levels of the averaged TD population, respectively.

\section{Statistical analysis}

Means with standard deviation were calculated for DTI parameters for the TD pediatric population and subjects with hydromyelia and syringomyelia. A standard least squares regression model based on restricted maximum likelihood (REML) was used to compare the DTI parameters between the TD population without any hydromyelia or syringomyelia $(n=22)$ and subjects with hydromyelia and syringomyelia $(n=4)$. This model was constructed by looking at the group differences between the TD population and subjects with hydromyelia and syringomyelia lesion by assuming ROI level and group (TD population/subjects with hydromyelia and syringomyelia lesion) composition were the fixed effects and the subjects modeled as a random effect. A $p$ value of 0.05 was used throughout to determine the statistical significance. There was no correction performed for multiple comparisons. All statistical analysis was performed using JMP pro 13.0 software.

\section{Diagnostic criteria for hydromyelia and syringomyelia}

Hydromyelia is diagnosed with a MRI scan and findings represent a fluid collection that follows cerebrospinal fluid signal on all pulse sequences and is located centrally within the SC and pathologically represents a dilatation of the central canal. Syringomyelia represents a similar appearing fluid collection, however is present off midline within the $\mathrm{SC}$ and therefore represents a fluid collection that is not a dilated central canal.

\section{Results}

The findings of hydromyelia and syringomyelia lesion were radiologically confirmed for all the subjects on the T2weighted MRI images (Fig. 1).

\section{Group analysis}

We observed significantly increased MD $(p=0.010)$ and significantly decreased $\mathrm{AD}(p=0.007)$ values in the cord above the highest lesion (Mid T3) compared to the TD population (Table 1). However, no significant difference in DTI parameters was observed in the cord below the lowest lesion (T10-T11) as compared to the TD population (Table 2).

\section{Single-subject analysis}

DTI parameters were significantly different in the cord above the syringomyelia lesion in subject 4 compared to the 

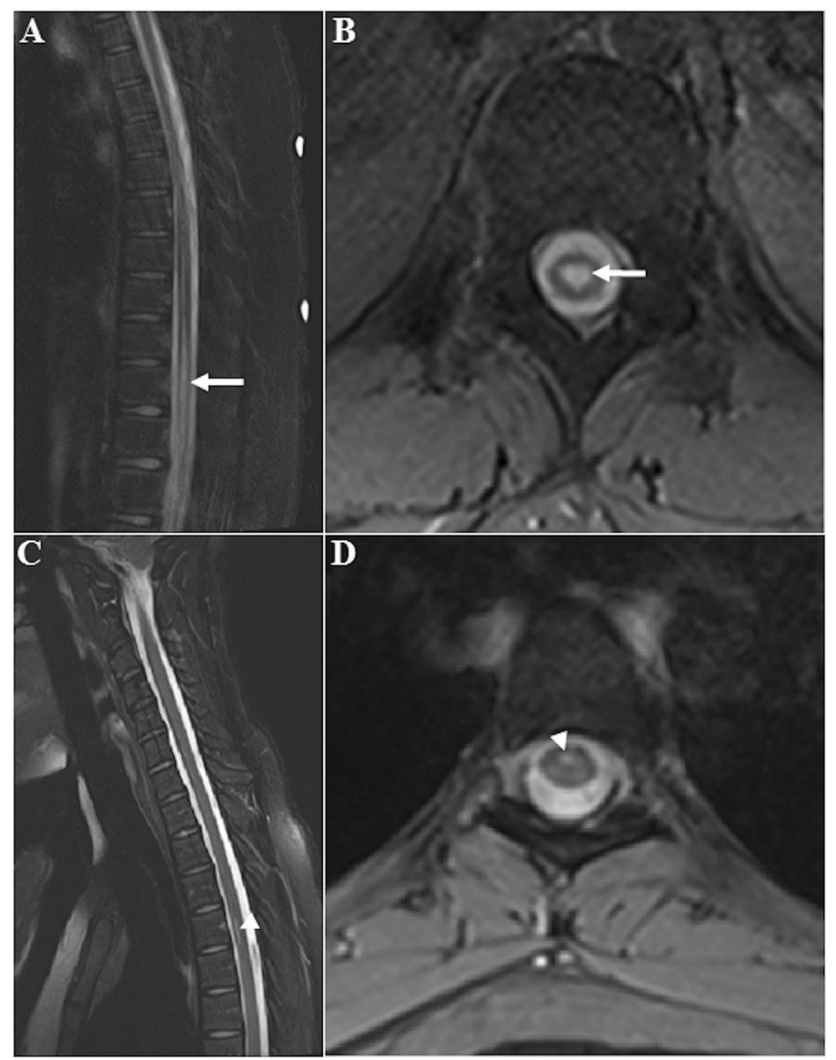

Fig. 1 Sagittal (a, c) and axial (b, d) T2-weighted MR images of a representative typically developing clinically normal subject with hydromyelia (white arrow) and syringomyelia (white arrowhead) lesions respectively. Axial T2-weighted images showing the hydromyelia lesion within the central canal which is at the center of the spinal cord while the syringomyelia lesion is anterior and to the right of the midline found except for a significant decrease in FA $(p=0.015)$ in subject 1 (Table 4$)$. In the cord below the syringomyelia lesion (subject 4), MD ( $p=0.0006)$ and $\mathrm{RD}(p=0.0003)$ were significantly increased while AD $(p=0.0066)$ was significantly decreased compared to TD population respectively (Table 4 ).

\section{Discussion}

To our knowledge, this study is the first to examine changes in the DTI parameters of the entire cord above and below the hydromyelia and syringomyelia lesion in the TD pediatric subjects with no known focal neurological deficits. Based on the literature, the incidence of hydromyelia and syringomyelia is uncommon to rare [9]. In our study of 26 TD pediatric subjects scanned, $16 \%$ had unexpected incidental findings of syringomyelia or hydromyelia. This increased incidence was unexpected in our TD population, however, it does not represent a selection bias. The TD subject selection was completely random and consisted of siblings of our spinal cord injury population and children of colleagues and family members. It was fortuitous that we were able to find the four TD subjects with these incidental findings. An apriori theory was that the cord above and below these congenital lesions should not be statistically different from the TD population. This is based on the lesions in this population which were incidental MR findings with no prior history of SC abnormality or focal neurologic findings. Also, a prior study by Yan et al. showed no

Table 1 DTI parameters of FA, MD, AD, and RD (group analysis) in normal appearing cord on MRI in the TD pediatric population and subjects with hydromyelia and syringomyelia above the highest lesion (Mid T3)

\begin{tabular}{lllll}
\hline & DTI parameters & & \\
\cline { 2 - 5 } & $\begin{array}{l}\text { FA (TD, } \\
\text { subjects) }\end{array}$ & $\begin{array}{l}\text { MD (TD, } \\
\text { subjects) }\end{array}$ & $\begin{array}{l}\text { AD (TD, } \\
\text { subjects) }\end{array}$ & $\begin{array}{l}\text { RD (TD, } \\
\text { subjects) }\end{array}$ \\
\hline TD vs. subjects with hydromyelia & $\begin{array}{l}0.57 \pm 0.09, \\
0.52 \pm 0.10 \\
\text { and syringomyelia }\end{array}$ & $\begin{array}{l}1.06 \pm 0.22, \\
1.08 \pm 0.25\end{array}$ & $\begin{array}{l}1.80 \pm 0.34, \\
1.73 \pm 0.38\end{array}$ & $\begin{array}{l}0.68 \pm 0.19, \\
0.75 \pm 0.23 \\
p=0.066\end{array}$ \\
\hline
\end{tabular}

Units for $\mathrm{MD}, \mathrm{AD}$, and $\mathrm{RD}$ are given in $10^{-3} \mathrm{~mm}^{2}$

$F A$ fractional anisotropy, $M D$ mean diffusivity, $A D$ axial diffusivity, $R D$ radial diffusivity, $T D$ typically developing, $D T I$ diffusion tensor imaging $* p$ Value $\leq 0.05$ indicates statistical significance

TD population (Table 3). In subject 4 the DTI values showed a significant decrease in FA $(p<0.0001)$, increase in $\mathrm{MD}(p<0.0001)$, decrease in $\mathrm{AD}(p<0.0001)$, and increase in $\mathrm{RD}(p<0.0001)$ above the syringomyelia lesion. However, no significant difference in DTI parameters were found in the cord above the hydromyelia lesion in subjects 1,2 , and 3 (Table 3 ). In the cord below the hydromyelia lesion, no significant difference in DTI parameters were
DTI abnormality in patients with syringomyelia lesions [10]. Our results however showed DTI cord abnormality above and below these lesion using both group and individual analysis. In group analysis, the cord above the lesion in all four subjects showed abnormal DTI metrics. Using single-subject analysis, we found that DTI parameters were significantly different in the cord above the syringomyelia lesion, while cord above the hydromyelia lesion showed no 
Table 2 DTI parameters of FA, MD, AD, and RD (group analysis) in normal appearing cord on MRI in the TD pediatric population and subjects with hydromyelia and syringomyelia below the lowest lesion (T10-T11)

\begin{tabular}{|c|c|c|c|c|}
\hline & \multicolumn{4}{|c|}{ DTI parameters } \\
\hline & $\begin{array}{l}\text { FA (TD, } \\
\text { subjects) }\end{array}$ & $\begin{array}{l}\text { MD (TD, } \\
\text { subjects) }\end{array}$ & $\begin{array}{l}\mathrm{AD}(\mathrm{TD} \\
\text { subjects) }\end{array}$ & $\begin{array}{l}\mathrm{RD}(\mathrm{TD} \\
\text { subjects) }\end{array}$ \\
\hline $\begin{array}{l}\text { TD vs. subjects with hydromyelia } \\
\text { and syringomyelia }\end{array}$ & $\begin{array}{l}0.46 \pm 0.09 \\
0.45 \pm 0.06 \\
p=0.594\end{array}$ & $\begin{array}{l}0.80 \pm 0.23 \\
0.88 \pm 0.27 \\
p=0.999\end{array}$ & $\begin{array}{l}1.20 \pm 0.31 \\
1.34 \pm 0.46 \\
p=0.829\end{array}$ & $\begin{array}{l}0.60 \pm 0.20 \\
0.65 \pm 0.18 \\
p=0.921\end{array}$ \\
\hline
\end{tabular}

Units for $\mathrm{MD}, \mathrm{AD}$, and $\mathrm{RD}$ are given in $10^{-3} \mathrm{~mm}^{2} / \mathrm{s}$

$F A$ fractional anisotropy, $M D$ mean diffusivity, $A D$ axial diffusivity, $R D$ radial diffusivity, $T D$ typically developing, $D T I$ diffusion tensor imaging $* p$ Value $\leq 0.05$ indicates statistical significance

Table 3 DTI parameters of FA, MD, AD, and RD (single-subject analysis) in normal appearing cord on MRI above the hydromyelia (subjects 1, 2, 3) and syringomyelia (subject 4) lesions and the TD pediatric population

\begin{tabular}{lllll}
\hline \multicolumn{5}{l}{ DTI parameters } \\
\cline { 2 - 5 } & $\begin{array}{l}\text { FA (TD, } \\
\text { subjects) }\end{array}$ & $\begin{array}{l}\text { MD (TD, } \\
\text { subjects) }\end{array}$ & $\begin{array}{l}\text { AD (TD, } \\
\text { subjects) }\end{array}$ & $\begin{array}{l}\text { RD (TD, } \\
\text { subjects) }\end{array}$ \\
\hline TD vs. & $0.57 \pm 0.09$, & $1.04 \pm 0.23$, & $1.77 \pm 0.36$, & $0.68 \pm 0.19$, \\
subject 1 & $0.61 \pm 0.09$ & $1.00 \pm 0.18$ & $1.81 \pm 0.37$ & $0.60 \pm 0.12$ \\
& $p=0.102$ & $p=0.432$ & $p=0.193$ & $p=0.754$ \\
TD vs. & $0.56 \pm 0.09$, & $1.04 \pm 0.24$, & $1.76 \pm 0.37$, & $0.68 \pm 0.20$ \\
subject 2 & $0.52 \pm 0.06$ & $1.09 \pm 0.18$ & $1.73 \pm 0.27$ & $0.77 \pm 0.17$ \\
& $p=0.953$ & $p=0.066$ & $p=0.268$ & $p=0.063$ \\
TD vs. & $0.57 \pm 0.09$, & $1.07 \pm 0.24$, & $1.82 \pm 0.36$, & $0.69 \pm 0.21$, \\
subject 3 & $0.53 \pm 0.07$ & $1.00 \pm 0.16$ & $1.65 \pm 0.29$ & $0.68 \pm 0.13$ \\
& $p=0.950$ & $p=0.980$ & $p=0.983$ & $p=0.975$ \\
TD vs. & $0.57 \pm 0.09$, & $1.05 \pm 0.22$, & $1.79 \pm 0.35$, & $0.68 \pm 0.19$, \\
subject 4 & $0.44 \pm 0.11$ & $1.10 \pm 0.42$ & $1.59 \pm 0.55$ & $0.85 \pm 0.36$ \\
& $* p<0.0001$ & $* p<0.0001$ & $* p<0.0001$ & $* p<0.0001$ \\
\hline
\end{tabular}

Units for $\mathrm{MD}, \mathrm{AD}$, and $\mathrm{RD}$ are given in $10^{-3} \mathrm{~mm}^{2} / \mathrm{s}$

$F A$ fractional anisotropy, $M D$ mean diffusivity, $A D$, axial diffusivity, $R D$ radial diffusivity, $T D$, typically developing, $D T I$ diffusion tensor imaging

${ }^{*} p$ Value $\leq 0.05$ indicates statistical significance

significant DTI differences compared to the TD population. In the cord below the syringomyelia lesion in subject 4, $\mathrm{MD}, \mathrm{AD}$, and RD were significantly different, using singlesubject analysis, compared to TD population. Additionally, using single-subject analysis, a subject with hydromyelia showed significant difference in FA in the cord below the lesion.

The use of DTI to quantitatively assess the microstructural changes in the clinically normal pediatric subjects with hydromyelia and syringomyelia has not been reported. Previous studies have evaluated cervical syringomyelia in adult patients using DTI $[16,17]$. Agosta et al. reported the presence of preserved white matter tracts around and beyond the syrinx despite the cervical syrinx visible on the T2-weighted image in one patient with cervical syringomyelia and multiple sclerosis [16]. Roser et al. found
Table 4 DTI parameters of FA, MD, AD, and RD (single-subject analysis) in normal appearing cord on MRI below the hydromyelia (subjects 1,2) and syringomyelia (subject 4) lesions and the TD pediatric population

\begin{tabular}{lllll}
\hline \multicolumn{5}{l}{ DTI parameters } \\
\cline { 2 - 5 } & $\begin{array}{l}\text { FA (TD, } \\
\text { subjects) }\end{array}$ & $\begin{array}{l}\text { MD (TD, } \\
\text { subjects) }\end{array}$ & $\begin{array}{l}\text { AD (TD, } \\
\text { subjects) }\end{array}$ & $\begin{array}{l}\text { RD (TD, } \\
\text { subjects) }\end{array}$ \\
\hline TD vs. & $0.55 \pm 0.11$, & $0.98 \pm 0.28$, & $1.64 \pm 0.43$, & $0.66 \pm 0.24$, \\
subject 1 & $0.50 \pm 0.10$ & $1.11 \pm 0.23$ & $1.79 \pm 0.42$ & $0.77 \pm 0.17$ \\
& $* p=0.015$ & $p=0.626$ & $p=0.900$ & $p=0.239$ \\
TD vs. & $0.46 \pm 0.09$, & $0.79 \pm 0.23$, & $1.19 \pm 0.30$, & $0.60 \pm 0.20$ \\
subject 2 & $0.48 \pm 0.03$ & $1.09 \pm 0.14$ & $1.71 \pm 0.23$ & $0.78 \pm 0.10$ \\
& $p=0.162$ & $p=0.587$ & $p=0.299$ & $p=0.599$ \\
TD vs. & $0.56 \pm 0.10$, & $1.00 \pm 0.28$, & $1.68 \pm 0.43$, & $0.67 \pm 0.24$ \\
subject 4 & $0.46 \pm 0.08$ & $1.05 \pm 0.43$ & $1.57 \pm 0.63$ & $0.78 \pm 0.35$ \\
& $p=0.156$ & $* p=0.0006$ & $* p=0.0066$ & $* p=0.0003$ \\
\hline
\end{tabular}

Units for $\mathrm{MD}, \mathrm{AD}$, and $\mathrm{RD}$ are given in $10^{-3} \mathrm{~mm}^{2} / \mathrm{s}$

$F A$ fractional anisotropy, $M D$ mean diffusivity, $A D$ axial diffusivity, $R D$ radial diffusivity, $T D$ typically developing, $D T I$ diffusion tensor imaging

$* p$ Value $\leq 0.05$ indicates statistical significance

that FA values were lower at the level of the syrinx and were normalized at the levels beyond the syrinx in six patients with small focal cervical syringomyelia [17].

Yan et al. investigated DTI parameters in pediatric patients (mean age, 14.6 years) with syringomyelia secondary to Chiari I malformation and found decreased FA values at the syrinx levels suggesting increased microstructural damage within the SC parenchyma at this area [10]. In our subjects with hydromyelia and syringomyelia, we did not examine the DTI characteristic of the congenital lesion itself since this would clearly yield abnormal DTI values due to volume averaging with the benign cerebrospinal fluid and adjacent cord tissue. Therefore, in this study, we looked at the cord above and below the congenital lesion and performed both group analysis and single-subject analysis. Yan et al. did a group analysis on 23 patients with syringomyelia and their results showed no evidence of FA change in the levels surrounding the syrinx cavity area i.e. above and below the syrinx as compared to the control 
group [10]. However, in our current study, we found significant $\mathrm{MD}$ and $\mathrm{AD}$ changes in the cord above the highest lesion in the group analysis of small number of subjects ( $n$ $=4$ ). In our study we obtained the DTI parameters of the entire continuous segments of the cervical and thoracic cord while Yan et al. limited their data collection to only the cervical cord area. Based on Yan et al. findings and the fact that we studied TD subjects with no known neurological deficits, we expected normal DTI values in the cord, however the cord above the lesion was significantly different than the TD population suggesting that microstructural changes could be found in the normal appearing MRI cord above the benign syrinxes. Significantly increased MD and decreased $\mathrm{AD}$ values in the cord above the congenital lesion may represent microscopic degeneration of the fiber tracts at the lesion site, causing more wallerian degeneration in the cord above the lesion than below the lesion, as we did not find significant DTI differences in the cord below the lesion using group analysis.

The increased use of MRI found that some patients had small syrinxes with few or no symptoms. These cases were termed as hydromyelia and found slit-like central cerebrospinal fluid signal fluid collections which were fully contained in the central canal [18]. Another study showed that those patients with hydromyelia had nonspecific symptoms and hardly any neurological deficits [19]. In theory, hydromyelia represents a more benign cystic abnormality in the cord compared with syringomyelia. It can also be difficult to differentiate between a small nonpathological dilatation of the central canal (hydromyelia) and a syringomyelia [20]. DTI values could be used to detect the changes in the SC due to the syrinx cavities beyond the abnormalities visible on MRI. Roser et al. performed single-subject analysis of six adult patients with cervical syringomyelia and found that FA values were lower at the level of syrinx but normalized outside the syrinx suggesting that this syrinx may not represent a syringomyelia but a hydromyelia might be present [17]. In addition to group analysis, in our study single-subject analysis was also performed. It is important to investigate at a single-subject level the DTI changes in the cord as this may have future clinical utility. In our single-subject analysis of TD pediatric subjects, we found that the cord above the syringomyelia lesion (subject 4), demonstrated a significant decrease in FA, AD and significant increase in MD and RD compared to TD population. These changes may be associated with demyelination and degeneration of neural tracts in the cord above the syringomyelia lesion, hence increased barriers reducing water diffusivity along the axons and increased water diffusivity in the plane perpendicular to axons $[21,22]$. Syringomyelia may have a different etiology, in comparison to hydromyelia, possibly representing a combination of congenital and acquired factors such as a subclinical prior demyelinating, traumatic, or infectious/inflammatory process. The cord above the hydromyelia lesion in subjects 1,2 , and 3 showed no significant differences in DTI parameters suggesting that hydromyelia is likely a more benign process than syringomyelia. However, in the cord below the hydromyelia lesion, no significant difference in DTI parameters was found except for FA in subject 1 . In the cord below the syringomyelia lesion, $\mathrm{MD}, \mathrm{AD}$, and $\mathrm{RD}$ were significantly different compared to the TD population. This suggests that microstructural tissue alterations might be found in the cord below both the syringomyelia and hydromyelia lesions. The clinical relevance of a syrinx in the normal subjects is not always clear. In this study, DTI abnormalities in the cord above and below the congenital benign appearing syrinxes suggest the presence of evolving SC pathology not seen on conventional MRI. The pathology of the SC above and below the syrinxes may be one of the mechanisms linking syrinx to the neurological signs or symptoms in future data population.

The limitation of this study is the low number of subjects with hydromyelia and syringomyelia lesion as these MR findings are uncommon to rare in a TD population with no known neurological deficits. Future work with large number of subjects will be needed to assess the DTI differences in the cord above and below these lesions.

\section{Conclusions}

Despite the small sample size in our study, there were statistically significant findings in the DTI metrics. Hence this study underlines the importance of DTI to obtain quantitative information of the pathological characteristics of the tissue beyond the abnormalities visible on conventional MRI and demonstrates that DTI may have the potential to be used as an imaging biomarker. Future research is needed to define the standardized age-matched TD DTI data sets as well as the DTI analysis of selected white matter tracts and finally the reproducibility of these data sets. The finding of DTI abnormalities in TD subjects with congenital syringomyelia have not been previously described and differ from previous studies that showed normal DTI parameters in syringohydromyelia in subjects with Chiari I malformations. This study suggests that subjects with syringohydromyelia should not be included in a DTI analysis of a normative data population.

Acknowledgements This work was supported by National Institute of Neurological Disorders of the National Institutes of Health under award number R01NS079635. 


\section{Compliance with ethical standards}

Conflict of interest The authors declare that they have no conflict of interest.

\section{References}

1. Johnston I, Teo C. Disorders of CSF hydrodynamics. Childs Nerv Syst. 2000;16:776-99.

2. Ball MJ, Dayan AD. Pathogenesis of syringomyelia. Lancet. 1972;2:799-801.

3. Sgouros S. Encyclopedia of neuroscience; 2009, 839-47.

4. Sgouros Spyros. Cerebrospinal fluid disorders; 2009, 318-36.

5. Milhorat TH. Classification of syringomyelia. Neurosurg Focus. 2009;8:E1.

6. Al-Shatoury HA. Syringomyelia. In: Benbadis Selim R, chief editor. eMedicine; 2012.

7. Sandoval-Garcia C, Iskandar BJ. Epidemiology of syringomyelia in children. The ISPN guide to pediatric neurosurgery.

8. Vandertop WP. Syringomyelia. Neuropediatrics. 2014;45:3-9.

9. American Syringomyelia and Chiari Alliance Project. The prevalence of SM in the US. Longview, TX: American Syringomyelia and Chiari Alliance Project; 2009.

10. Yan H, Zhu Z, Liu Z, Zhang X, Sun X, Sha S, et al. Diffusion tensor imaging in cervical syringomyelia secondary to Chiari I malformation: preliminary results. Spine (Phila $\mathrm{Pa}$ 1976). 2015;40:E381-7.

11. Barakat N, Mohamed FB, Hunter LN, Shah P, Faro SH, Samdani $\mathrm{AF}$, et al. Diffusion tensor imaging of the normal pediatric spinal cord using an inner field of view echo-planar imaging sequence. AJNR Am J Neuroradiol. 2012;33:1127-33.

12. Finsterbusch J. Improving the performance of diffusion-weighted inner field-of-view echo-planar imaging based on 2D-selective radiofrequency excitations by tilting the excitation plane. J Magn Reson Imaging. 2012;35:984-92.
13. Saksena S, Middleton DM, Krisa L, Shah P, Faro SH, Sinko R, et al. Diffusion tensor imaging of the normal cervical and thoracic pediatric spinal cord. AJNR Am J Neuroradiol. 2016;37:2150-57.

14. Middleton DM, Mohamed FB, Barakat N, Hunter LN, Shellikeri $\mathrm{S}$, Finsterbusch J, et al. An investigation of motion correction algorithms for pediatric spinal cord DTI in healthy subjects and patients with spinal cord injury. Magn Reson Imaging. 2014;32:433-9.

15. Chang LC, Jones DK, Pierpaoli C. RESTORE: Robust estimation of tensors by outlier rejection. Magn Reson Med. 2005;53:1088-95.

16. Agosta F, Rovaris M, Benedetti B, Valsasina P, Filippi M, Comi G. Diffusion tensor MRI of the cervical cord in a patient with syringomyelia and multiple sclerosis. J Neurol Neurosurg Psychiatry. 2004;75:1647.

17. Roser F, Ebner FH, Maier G, Tatagiba M, Nagele T, Klose U. Fractional anisotropy levels derived from diffusion tensor imaging in cervical syringomyelia. Neurosurgery. 2010;67:901-5.

18. Holly LT, Batzdorf U. Slitlike syrinx cavities: a persistent central canal. J Neurosurg Spine. 2002;97:161-5.

19. Roser F, Ebner FH, Danz S, Riether F, Ritz R, Dietz K, et al. Three-dimensional constructive interference in steady-state magnetic resonance imaging in syringomyelia: advantages over conventional imaging. J Neurosurg. 2008;8:429-35.

20. Roser F, Ebner FH, Sixt C, Hagen JM, Tatagiba MS. Defining the line between hydromyelia and syringomyelia. A differentiation is possible based on electrophysiological and magnetic resonance imaging studies. Acta Neurochir (Wien). 2010;152:213-9.

21. Zhang J, Jones M, DeBoy CA, Reich DS, Farrell JA, Hoffman $\mathrm{PN}$, et al. Diffusion tensor magnetic resonance imaging of Wallerian degeneration in rat spinal cord after dorsal root axotomy. $\mathrm{J}$ Neurosci. 2009;29:3160-71.

22. Cohen-Adad J, Leblond H, Delivet-Mongrain H, Martinez M, Benali H, Rossignol S. Wallerian degeneration after spinal cord lesions in cats detected with diffusion tensor imaging. Neuroimage. 2011;57:1068-76. 